\title{
Nonequilibrium pattern formation in strongly interacting driven colloids
}

\author{
Hartmut Löwen* and Joachim Dzubiella
}

Institut für Theoretische Physik II, Heinrich-Heine-Universität Düsseldorf, Universitätsstraße 1, D-40225, Düsseldorf, Germany.

E-mail: hlowen@thphy.uni-duesseldorf.de

Received 21st March 2002, Accepted 7th June 2002

First published as an Advance Article on the web 27th September 2002

\begin{abstract}
Dynamical instabilities are discussed for strongly interacting colloidal suspensions which are driven into nonequilibrium by an external field in the limit where hydrodynamic interactions can be neglected. Brownian dynamics computer simulations indicate that stripe-like patterns of particles driven alike are spontaneously formed if the external drive exceeds a critical strength. Recent previous studies of stripe formation obtained for symmetric equimolar mixtures in the steady state are reviewed. These results are then extended in two directions: first, we show that stripe-like segregation occurs also in asymmetric mixtures and observe an additional compression/expansion effect in the stripes composed of the small/large particles. Second, we study the relaxation into the stripepatterned steady state starting from a uniform demixed state and show that different transient processes such as jamming, anisotropic coarsening and void formation are relevant on the route into the stratified steady state.
\end{abstract}

\section{Introduction}

Almost fifty years ago, it was realized by Whitmore ${ }^{1}$ that adding a second colloidal component to a sedimenting suspension with a different buoyant mass causes an increase in the settling speed. This finding can be attributed to an instability with respect to vertical density variations which was macroscopically confirmed by Weiland et al. ${ }^{2}$ and classified by Batchelor and van Rensburg. ${ }^{3}$ A closer inspection of the patterns induced via the instability reveals that these are very complex and complicated structures which are mainly generated by the hydrodynamic backflow (for other more recent backflow effects during sedimentation, see ref. 4). Hydrodynamic interactions between the different sedimenting particles are indeed the clue to explain the physical origin of this instability. Namely the instability even occurs at low particle volume fractions where the particles are noninteracting via direct interaction forces.

In this discussion paper, we study a complementary case of strongly interacting colloids without hydrodynamic interactions in an external driving field as e.g. gravity. The motivation to do so is twofold: first, such an extreme situation is realized in highly charged and strongly deionized suspensions which exhibit strong correlations even at very low colloidal volume fractions where hydrodynamic interactions do not play a dominant role. Second, more fundamentally, in the absence of any hydrodynamic flow effect, the physics is expected to be simpler such that a full characterization of the dynamical instabilities becomes feasible. In fact, for a highly correlated system, the thermal energy is small compared to a typical interaction energy such that a dynamical 
instability can be simply understood by a competition of two effects: the internal interaction forces between the particles and the external driving force. Once the situation is clarified, one may add hydrodynamic interactions at a later stage to check their influence on nonequilibrium pattern formation.

In order to keep the situation simple, we restrict ourselves to two-component mixtures which are driven by different but constant (i.e. space- and time-independent) forces. One immediate realization is gravity but an electric field or gradients of laser-optical fields are also conceivable. Our studies here are completely based on nonequilibrium Brownian dynamics computer simulations. We find a nonequilibrium segregation transition from a uniform mixed state to a nonuniform state characterized by a stripe-like pattern of particles driven alike.

Similar stripe-like nonequilibrium segregation phenomena have been observed experimentally and studied theoretically in different systems, all of them are qualitatively different from our situation:

(i) As already discussed, in hydrodynamically driven low-density suspensions, the hydrodynamic interactions enforce the instability which could then be even perpendicular to the drive. ${ }^{2,3,5-8}$

(ii) In granular matter and particle-gas suspensions, there is a significant inertia which is negligible for colloids. Stripe-like segregation phenomena under shear and/or gravity have been detected in various publications. ${ }^{9-12}$

(iii) In pedestrian dynamics, similar models are used, most of them involve an inertia and are thus different from the completely overdamped Brownian dynamics of colloids. The stripe-like pattern formation has the intuitive meaning of lane formation in pedestrian zones. ${ }^{13-15}$

The paper is organized as follows: In section II, we describe the general model and the simulation technique used. In section III, we review recent results obtained for the nonequilibrium lane formation process. Original results are contained in section IV and V where the case of asymmetric mixtures is studied and the relaxation into the steady state involving stripe-patterns is investigated. We find a compression effect in the lanes consisting of small particles and an expansion effect in those lanes possessing big particles and identify different transient structures such as voids, jammed states and anisotropic coarsening on the way into the completely stratified steady state. We finish with remarks on an experimental verification in section VI and give a list of open questions in section VII.

\section{The model and the simulation technique}

In our model, ${ }^{16}$ we consider an asymmetric binary colloidal mixture comprising $N_{1}+N_{2}$ Brownian colloidal particles in an area $S$ (i.e. in $d=2$ spatial dimensions). $N_{1}$ particles are of type 1 , the other $N_{2}$ are of type 2 with partial number densities $\rho_{1}=N_{1} / S$ and $\rho_{2}=N_{2} / S$. The colloidal suspension is held at a fixed temperature $T$ via the bath of microscopic solvent particles. Two colloidal particles interact via a set of effective Yukawa pair potentials

$$
V_{i j}(r) / k_{\mathrm{B}} T=U_{0} \sigma_{i j} \exp \left[-\kappa\left(r-\sigma_{i j}\right)\right] / r,
$$

where $(i j)=(11),(12),(22)$. Here $r$ is the center-to-center separation, $U_{0}$ is the interaction strength measured in terms of the thermal energy $k_{\mathrm{B}} T$ and $\kappa$ is the inverse screening length.

The set of diameters, $\sigma_{i j}$, is additive and given by

$$
\begin{gathered}
\sigma_{11}=\sigma \\
\sigma_{22}=q \sigma_{11}=q \sigma \\
\sigma_{12}=\left(\sigma_{11}+\sigma_{22}\right) / 2
\end{gathered}
$$

In the following, $\sigma$ will serve as a length scale. The screened Coulomb interaction models bidisperse charge-stabilized suspensions confined to two dimensions. ${ }^{17-19}$

The dynamics of the colloids is completely overdamped Brownian motion. The friction constant is $\xi^{(j)}=3 \pi \eta \sigma_{j j}(j=1,2)$ with $\eta$ denoting the shear viscosity of the solvent. The constant external force acting on the $i$ th particle of species $j, \vec{F}_{i}^{(j)}$, has the same direction but different amplitude for the both constituents of the binary mixture. It is $\vec{F}_{i}^{(1)}=F^{(1)} \vec{e}_{y}$ and $\vec{F}_{i}^{(2)}=F^{(2)} \vec{e}_{y}$ where $\vec{e}_{y}$ is a unit vector along the $y$-direction of the system. 
The stochastic Langevin equations for the colloidal trajectories $\vec{r}_{i}^{(j)}(t)(j=1,2)$ (with $i=1, \ldots, N_{1}$ for $j=1$ and $i=1, \ldots, N_{2}$ for $j=2$ ) read as

$$
\begin{aligned}
\xi^{(j)} \frac{\mathrm{d} \vec{r}_{i}^{(j)}}{\mathrm{d} t}= & -\vec{\nabla}_{\vec{r}_{i}^{(j)}}\left[\sum_{k=1}^{N j^{\prime}} V_{j j^{\prime}}\left(\left|\vec{r}_{i}^{(j)}-\vec{r}_{k}^{\left(j^{\prime}\right)}\right|\right)\right. \\
& \left.+\sum_{k=1, k \neq i}^{N_{j}} V_{j j}\left(\left|\vec{r}_{i}^{(j)}-\vec{r}_{k}^{(j)}\right|\right)\right]+\vec{F}_{i}^{(j)}+\vec{K}_{i}^{(j)}(t),
\end{aligned}
$$

where $j^{\prime}$ is the complementary index to $j\left(j^{\prime}=1\right.$ if $j=2$ and $j^{\prime}=2$ if $j=1$ ). The right-hand-side includes all forces acting on the colloidal particles, namely the force resulting from inter-particle interactions, the external constant force, and the random forces $\vec{K}_{i}^{(j)}$ describing the collisions of the solvent molecules with the $i$ th colloidal particle of species $j$. The latter are Gaussian random numbers with zero mean, $\vec{K}_{i}^{(j)}=0$, and variance

$$
\overline{\left(\vec{K}_{i}^{(k)}\right)_{\alpha}(t)\left(\vec{K}_{j}^{(n)}\right)_{\beta}\left(t^{\prime}\right)}=2 k_{\mathrm{B}} T \xi^{(j)} \delta_{\alpha \beta} \delta_{i j} \delta_{k n} \delta\left(t-t^{\prime}\right) .
$$

The subscripts $\alpha$ and $\beta$ stand for the two Cartesian components. Note that within this simple Langevin picture, hydrodynamic interactions are ignored.

We solve the Langevin equations of motion by Brownian dynamics simulations ${ }^{20-22}$ using a finite time-step and the technique of Ermak. ${ }^{23,24}$ We use a square cell of length $\ell$ with periodic boundary conditions. The typical size of the time-step was $0.003 \tau_{\mathrm{B}}$, where $\tau_{\mathrm{B}}=\xi^{(1)} \sigma^{2} / U_{0} k_{\mathrm{B}} T$ is a suitable Brownian timescale. We simulated typically $2 \times 10^{4}$ time steps which corresponds to a simulation time of $60 \tau_{\mathrm{B}}$.

\section{Review of recent studies}

Previous studies have focused on the steady state of an equimolar symmetric mixture. In this case, $N_{1}=N_{2} \xi^{(1)}=\xi^{(2)}=\xi$ and $V_{11}(r)=V_{22}(r)=V_{12}(r)=V(r)$. Using extensive Brownian dynamics computer simulation studies, Hoffmann and co-authors ${ }^{16}$ have found that a strongly interacting suspension exhibits lane formation if the external drive $\left|F^{(2)}-F^{(1)}\right|$ exceeds a critical value. The lanes involve particles either of type 1 or of type 2 which are sliding against each other in the field direction. In the lane involving 1 particles only, all these particle are drifting with a global velocity $F^{(1)} \vec{e}_{y} / \xi$ while opposite regions which involve 2 particles are streaming with the overall velocity $F^{(2)} \vec{e}_{y} / \xi$. The width of the lanes perpendicular to the drive direction involves several particle layers and is comparable to the size of the simulation box. This implies that lane formation is a macrophase separation. By subtracting the overall velocity using a Galilei transformation one readily sees that within the completely separated lanes, equilibrium Boltzmann statistics is realized. The system just separates into two different equilibrium states which are drifting relative to each other. In reducing the external drive one observes a significant hysteresis indicating that lane formation is a nonequilibrium first-order transition. A simple phenomenological theory describing lane formation as a competition between pair interaction forces and the external force was also proposed in ref. 16 . This theory predicted the trends correctly for varying particle density, for varying temperature and for different ranges $1 / \kappa \sigma$ of the interparticle interactions.

We further remark that lane formation is a very general and robust phenomenon: it was shown ${ }^{16}$ to occur also in three spatial dimensions, and the qualitative features do not depend on details of the interparticle interactions. The external field can also be slowly oscillating in time without destroying formation of lanes.

Just below the critical strength needed to generate lane formation, there is a precursor of anisotropic coarsening which is presumably second order and involves stripe-like patterns (or "worms") with a width of one single particle perpendicular to the field while they are quite extended in the $y$-direction parallel to the field. This has been detected by calculating strongly anisotropic structural correlations in further Brownian dynamics computer simulations by Chakrabarti and co-authors. ${ }^{25}$ Furthermore it can be understood as a dynamical instability from an instability analysis of a dynamical density functional theory. This theory yields quantitative agreement with the simulation data. The physical picture arising for lane formation is a 
competition of two effects: first, entropy of mixing would like to destabilize any $1-2$-interface and generates a mixed uniform state for small external fields. On the other hand, field-induced collisions between 1 and 2 particles amplify a lateral segregation between different particle species, an effect which is dominant for large driving strengths.

As lane formation occurs for highly correlated systems it is tempting to ask whether it also happens for colloidal solids. In two spatial dimensions, for a symmetric mixture without any drive, stable solids possess a randomly occupied hexagonal lattice structure. In ref. 26 it was shown that, under the influence of an external driving field, the solid exhibits a reentrant freezing phenomenon. It first melts mechanically via the external field and then recrystallizes into two phase separated 1 and 2 solids using a similar lane formation process in the solid phase.

Let us now briefly discuss the effect of relative tilted external forces, i.e. the case of non-parallel $\vec{F}^{(1)}$ and $\vec{F}^{(2)}$. Based on general reasoning and computer simulations, it was shown in refs. 26 and 27 that lane formation does also occur for external forces tilted relative to each other. The direction of the lanes, however, is now parallel to the difference vector $\vec{F}^{(2)}-\vec{F}^{(1)}$. Finally let us mention a recent simulation study of Ramaswamy et al. ${ }^{28}$ with non-symmetric interactions (i.e. $V_{12}(r) \neq$ $\left.V_{11}(r)=V_{22}(r)\right)$ which revealed nontrivial nonequilibrium melting and freezing cycles.

\section{Asymmetric mixtures}

In this section, we show that lane formation also occurs in asymmetric mixtures. Our simulation studies involve the following parameter combinations for the model proposed in section II: $N_{1}=100$ large particles and $N_{2}=500$ small particles are in a square box. The total particle number density is $\rho \sigma^{2}=1.5$. The parameters for the Yukawa pair potential are: $U_{0}=2.5$ and $\kappa \sigma=4$. The size asymmetry $q=\sigma_{22} / \sigma_{11}$ is 0.5 .

Four simulation snapshots in the steady state for increasing external forces are shown in Fig. 1. While in Fig. 1(a) an equilibrium snapshot of a demixed uniform is shown in the absence of any drive, an increasing drive forces the system into a stripe-like structure which becomes more pronounced the larger the drive is. For strong drive (Fig. 1(d)), there is clear separation between big and small particles, both of them are contained in lanes.

Although the formation of lanes is qualitatively similar to the case of symmetric mixtures, there is an additional effect visible in the snapshot of Fig. 1(d): The lanes involving the small particles are compressed while the lanes involving the big particles are more dilute as compared to their mixed state (see Fig. 1(a)). This is due to the fact that a driven big particle excludes small particles from a "tube" around it. We speculate that one may even achieve a phase separation where the small particles undergo a freezing transition in their lanes.

\section{Relaxation into the steady state}

If the external field is suddenly turned on, the system relaxes from an initial homogeneous mixed phase into a phase-separated phase provided the field strength is large enough. We have investigated the transient dynamics of the relaxation process via Brownian dynamics computer simulations involving $N_{1}+N_{2}=500$ particles. The system was modeled to be an equimolar symmetric mixture with interaction parameters $U_{0}=2.5$ and $\kappa \sigma=4$ with a total density of $\rho \sigma^{2}=1.0$. The magnitude of $\left|F^{(2)}-F^{(2)}\right| \sigma / k_{\mathrm{B}} T$ was 150 well beyond the critical force to get lane formation. ${ }^{16}$ The external field was turned on at a time $t=0$. Four snapshots taken at four different times $0,0.1 \tau_{\mathrm{B}}$, $\tau_{\mathrm{B}}$, and $10 \tau_{\mathrm{B}}$ are presented in Fig. 2. In Fig. 2(a), the disordered completely mixed starting configuration is shown. After turning on the field, two typical snapshots on the route towards lane formation are shown in Fig. 2(b) and 2(c). Qualitatively, first of all one recognizes the existence of voids which are transient structures as they disappear again in the relaxed steady state of fully developed lanes, see Fig. 2(d). Furthermore there are two other transient effects: in Fig. 2(b), jamming states are visible. Three examples of regions where jamming occur are indicated by the circles in Fig. 2(b). These consist of oppositely driven fronts of the same particles which block each other for a considerable amount of time. The jamming states are extended perpendicular to the field. Although these jamming states have quite a long life-time, they are not stable in our study. For dynamics in a confined strip, as e.g. realized in a pedestrian zone, these jamming states survive 
(a)

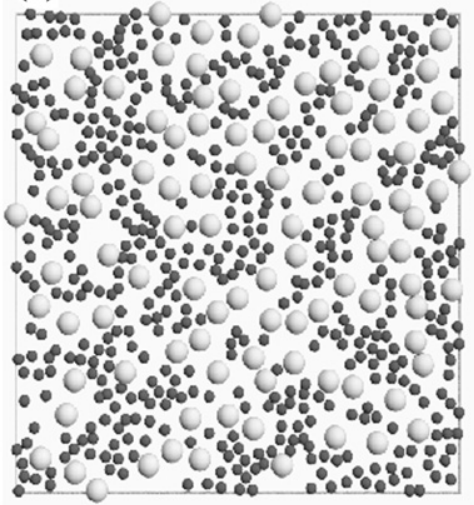

(c)

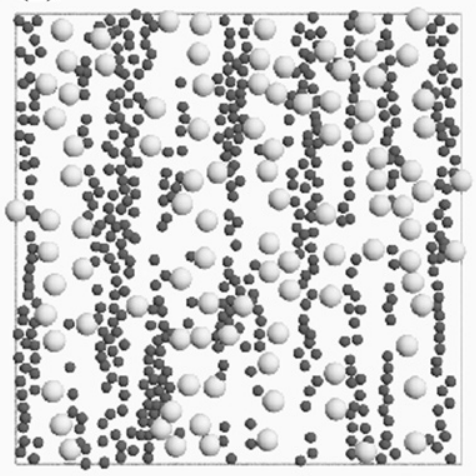

(b)

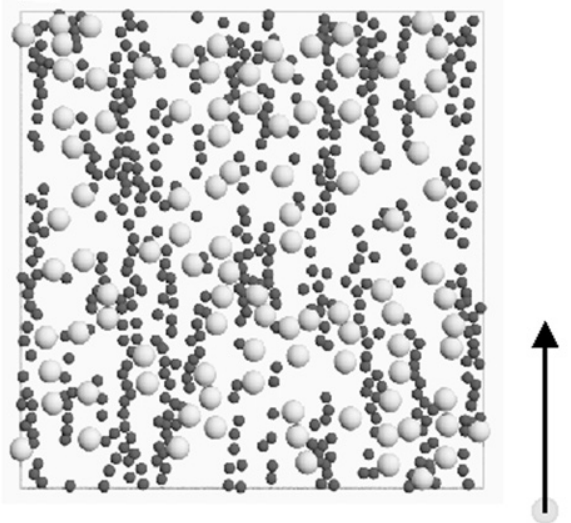

(d)

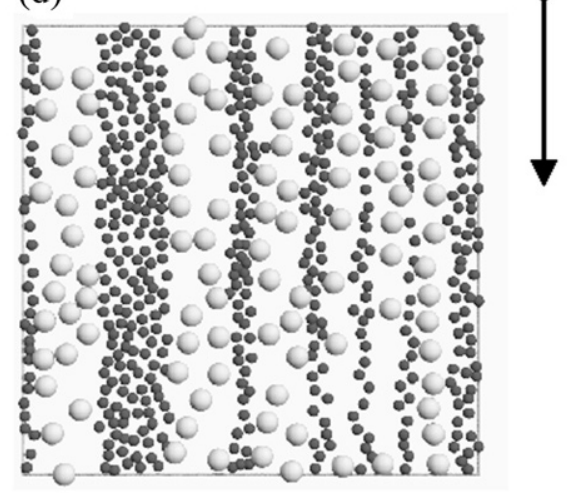

Fig. 1 Simulation snapshots for a driven asymmetric mixture. The big particles are light grey spheres while the small particle are dark grey spheres. The parameters for the reduced driving forces $f^{(1)}=F^{(1)} \sigma / k_{\mathrm{B}} T$ and $f^{(2)}=F^{(2)} \sigma / k_{\mathrm{B}} T$ are: (a) $f^{(1)}=f^{(2)}=0$, (b) $f^{(1)}=f^{(2)}=10$, (c) $f^{(1)}=f^{(2)}=50$, (d) $f^{(1)}=f^{(2)}=100$.

for ever and trigger the "freezing by heating" transition found by Helbing and coworkers. ${ }^{13}$ Finally, one can clearly see stripe-like structures of finite width in both directions: the width perpendicular to the field is about one particle diameter, while the lengths of the structure parallel to the field are much greater. We call this structure anisotropic coarsening. Its direction is perpendicular to the jamming configuration. This structure becomes more and more persistent as time moves on, compare Fig. 2(b) with 2(c) for instance, and provides an ideal channel via which the completely demixed steady state is achieved, see Fig. 2(d). Having the instability analysis of ref. 25 in mind, we think that the formation of "worms" is the most efficient channel towards lane formation.

\section{Experimental verification}

The instability discussed here occurs for strongly interacting particles even in the absence of hydrodynamic interactions and has, to the best of our knowledge, not yet been detected in experiments on colloidal suspensions. Particular candidates for such an experimental verification are highly charged and strongly deionized bidisperse charged suspensions with a small volume fraction such that hydrodynamic interactions do not play a dominant role. The detection of the lanes may be difficult in scattering experiments in three dimensions but should be easier in confined suspensions whose trajectories can be followed in real-space and real-time. As far as possible external fields are concerned one can think about gravity first, or about electric fields and magnetic 
(a)

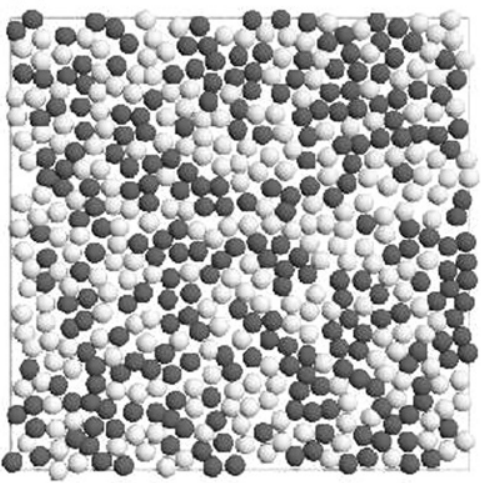

(c)

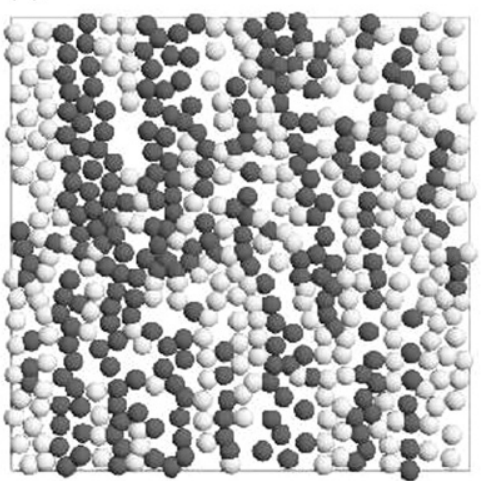

(b)

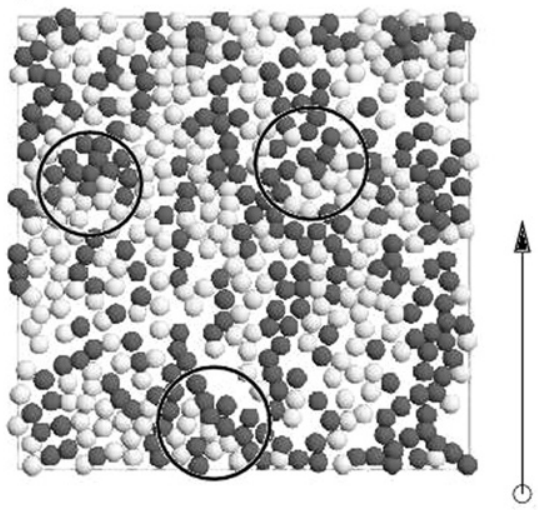

(d)

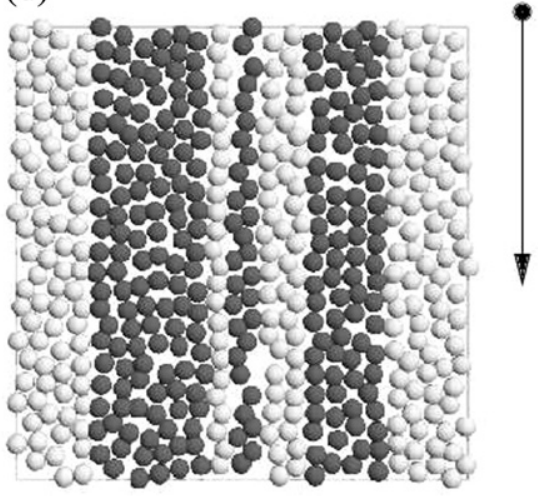

Fig. 2 Simulation snapshots for a driven symmetric mixture. The light grey spheres are pulled upwards while the dark grey spheres are pulled downwards. The relaxation from an initially mixed state at $t=0$ is shown for four different times: (a) $t=0$, (b) $t=0.1 \tau_{\mathrm{B}}$, (c) $t=\tau_{\mathrm{B}}$, (d) $t=10 \tau_{\mathrm{B}}$. The regions indicated by the three circles in (b) show jamming of the particles, which is a characteristic feature at the beginning of the relaxation.

fields. In the latter case, the colloidal particle should possess a magnetizable core. Once the transition has been found experimentally, it would further be interesting to compare quantitatively the location of the transition with the simulation predictions.

\section{Some open problems}

We end with a list of open problems.

First, it might be interesting to investigate in more detail the limit of very low densities. A reentrant behaviour is expected: for constant external field but increasing density, the system should exhibit two transitions from homogeneous state towards lane formation and then back to the homogeneous state.

Second, the extreme limit of very strongly asymmetric mixtures would be interesting to study in an external field. The question is whether the small particles will after all penetrate in the lanes formed by the big particles.

Third, on a next level of complexity, it would be interesting to put a phase-separating mixture into an external field. Then there is competition between fluid-fluid demixing driven by equilibrium thermodynamics and lane formation driven by the external field.

Finally it would be interesting to study the formation of worms more extensively. Finite system size effects have to be analyzed in detail and the effect of periodic boundary conditions on the lane formation in the simulation is not known exactly. Another scenario is a slowly growing 
worm-length which diverges at the precursor similar to a percolation transition. In order to clarify this in detail, more simulation studies and more microscopic and phenomenological theories are needed.

\section{Acknowledgements}

We thank T. Palberg, J. Chakrabarti, J. Sherwood and E. Allahyarov for helpful remarks. Financial support from the DFG (Sonderforschungsbereich 237) is gratefully acknowledged.

\section{References}

1 R. L. Whitmore, Brit. J. Appl. Phys., 1955, 6, 239.

2 R. H. Weiland, Y. P. Fessas and B. V. Ramaro, J. Fluid. Mech., 1984, 142, 383.

3 G. K. Batchelor and R. W. J. van Rensburg, J. Fluid. Mech., 1986, 166, 379.

4 P. N. Segré, F. Liu, P. Umbanhowar and D. A. Weitz, Nature, 2001, 409, 594.

5 R. G. Cox, Int. J. Multiphase Flow., 1990, 16, 617.

6 H. A. Nasr-El-Din, J. H. Masliyah and K. Nandakumar, Can. J. Chem. Eng., 1999, 11, 1003.

7 Y. Yan and J. H. Masliyah, Int. J. Multiphase Flow, 1993, 19, 875.

8 P. M. Biesheuvel, H. Verweij and V. Breedveld, AIChE J., 2001, 47, 45.

9 S. B. Santra, S. Schwarzer and H. Herrmann, Phys. Rev. E, 1996, 54, 5066.

10 P. Valiveti and D. L. Koch, Appl. Sci. Res., 1998, 58, 275.

11 P. Valiveti and D. L. Koch, Phys. Fluids, 1999, 11, 3283.

12 S. McNamara, E. G. Flekkoy and K. J. Maloy, Phys. Rev. E, 2000, 61, 4054.

13 D. Helbing, I. J. Farkas and T. Vicsek, Phys. Rev. Lett., 2000, 84, 1240.

14 D. Helbing, P. Molnár, I. J. Farkas and K. Bolay, Environ. Planning B: Planning Design, 2001, $28,361$.

15 C. Burstedde, K. Klauck, A. Schadschneider and J. Zittartz, Physica A, 2001, 295, 507.

16 J. Dzubiella, G. P. Hoffmann and H. Löwen, Phys. Rev. E, 2002, 65, 21402.

17 E. Chang and D. W. Hone, Europhys. Lett., 1988, 5, 635.

18 H. Löwen, J. N. Roux and J.-P. Hansen, J. Phys.: Condens. Matter, 1991, 3, 991.

19 H. Löwen, J. Phys.: Condens. Matter, 1992, 4, 10105.

20 G. P. Hoffmann and H. Löwen, Phys. Rev. E, 1999, 60, 3009.

21 G. P. Hoffmann and H. Löwen, J. Phys.: Condens. Matter, 2000, 12, 7359.

22 H. Löwen, J.-P. Hansen and J. N. Roux, Phys. Rev. A, 1991, 44, 1169.

23 M. P. Allen and D. J. Tildesley, Computer Simulations of Liquids, Clarendon Press, Oxford, 1989.

24 D. L. Ermak, J. Chem. Phys, 1975, 62, 4189.

25 J. Chakrabarti, J. Dzubiella and H. Löwen, 2002, to be published.

26 J. Dzubiella and H. Löwen, J. Phys.: Condens. Matter, 2002, to be published.

27 O. Biham and A. A. Middleton, Phys. Rev. A, 1992, 42, R6124-R6127.

28 M. Das, S. Ramaswamy and G. Ananthakrishna, Europhys. Lett., 2002, to be published. 